\title{
OPE of the stress tensors and surface operators
}

\author{
Xing Huang, ${ }^{a}$ Ling-Yan Hung ${ }^{b, c}$ and Feng-Li Lin ${ }^{a}$ \\ ${ }^{a}$ Department of Physics, National Taiwan Normal University, \\ Taipei, 116, Taiwan \\ ${ }^{b}$ Department of Physics and Center for Field Theory and Particle Physics, \\ Fudan University, Shanghai 200433, China \\ ${ }^{c}$ Collaborative Innovation Center of Advanced Microstructures, \\ Fudan University, Shanghai, 200433, China \\ E-mail: xingavatar@gmail.com, lyhung@fudan.edu.cn, \\ linfengli@phy.ntnu.edu.tw
}

ABSTRACT: We demonstrate that the divergent terms in the OPE of a stress tensor and a line (co-dimension two) operator of general shape in three dimensional spacetime cannot be constructed only from local geometric data depending only on the shape of the line. We verify this holographically for Wilson line operators or equivalently the twist operator corresponding to computing the entanglement entropy using the Ryu-Takayanagi formula. We discuss possible implications of this result.

Keywords: Wilson, 't Hooft and Polyakov loops, Gauge-gravity correspondence, AdSCFT Correspondence, Space-Time Symmetries

ARXIV EPRINT: 1502.02487 


\section{Contents}

1 Introduction 1

2 Ward identities and OPEs 3

2.1 Constructing expectation value of the stress tensor relating to OPE 4

3 Checking the circle in a conformal field theory 6

3.1 Hitting a rock - Failing Ward identity by local OPE for general entangling surfaces

3.1.1 Evidence 1

3.1.2 Evidence 2

4 Holographic calculation

4.1 Planar entangling surface perturbed

4.2 Holographic computation of the stress tensor

5 Conclusion

A Useful Identities regarding the local geometrical quantities

B Holographic stress tensor in the presence of twist operator

\section{Introduction}

Global symmetries and the Ward identities that follow are extremely important in field theories. It gives stringent constraints to the allowed structure of correlation functions. In turn, given knowledge of how fields should transform under a symmetry transformation, this information has to be stored in the OPE coefficients between the global symmetry charge operators and the field operator under consideration. These OPE coefficients between charge operators and local operators have been studied extensively. To take the simplest example, in a $1+1$ dimensional CFT, the OPE between the stress tensor and a primary scalar operator takes the following form:

$$
T_{z z}(z) \mathcal{O}_{\Delta}(0) \sim \frac{\Delta \mathcal{O}_{\Delta}}{z^{2}}+\frac{\partial \mathcal{O}_{\Delta}}{z}+\cdots
$$

where $\Delta$ is the (holomorphic) conformal dimension of the operator $\mathcal{O}_{\Delta}$. The first term stores data of how the scalar operator transforms under scaling transformation, while the second term describes how the scalar operator transforms under translation. One naturally wonders how the story generalizes to OPE's of charges with surface operators. For starter, it is certainly the case that Ward identities still apply whenever there is a global 
symmetry. If a global symmetry acts locally, it is natural to expect that a local charge density operator can be defined, and that the OPE coefficients - in this case coefficients of terms divergent in the inverse of the perpendicular distance of the charge density operator from the surface operator - should again carry information about how the surface operator transforms under the corresponding symmetry. These considerations should also have generalizations in studying higher symmetries [1] which necessarily involve symmetry transformation of higher dimensional objects. OPE coefficients between the stress tensor and a surface operator is a very good starting point to study these questions, particularly so in a CFT theory. The stress tensor exists in any CFT and its expectation value is very strongly constrained by conformal symmetry, which allows us to make guesses of the form of these coefficients. Moreover, for co-dimension two operators wrapping spherical regions for example, the form of the expectation values of the stress tensor is constrained down to only a single coefficient, thus allowing us to make quantitative checks of our guesses.

Co-dimension two operators are also very interesting for their connections to our understanding of entanglement entropy. The entanglement entropy and its cousins - the Rényi entropy- hold important information about the underlying field theory. It is known that the value of the entanglement entropy can be understood as the expectation value of a twist operator that introduces branch cuts over the entangling surface surrounding the region under consideration [2-5]. An understanding of these OPE coefficients of the stress tensor with the twist operators would allow us to compute at ease the entanglement entropy with entangling surfaces deformed arbitrarily from better understood ones, such as planar and spherical entangling surfaces. Ward identities of surface operators, particularly those involving entanglement entropy, have been considered before [6, 7]. More recently there is a flurry of work towards understanding the entanglement entropy of more general shapes at least perturbatively from spherical/planar ones [8-13]. Our work is the first attempt towards understanding these perturbative changes via OPE coefficients.

Our strategy can be summarized as follows. We insert the stress tensor at a perpendicular distance $\epsilon$ from the surface operator. As in $[14,15]$ we would like to focus on the ratio of the expectation value of the stress tensor to that of the surface operator alone. Simply from dimensional analysis, it is expected that the leading term of the OPE contains a $1 / \epsilon^{d}$ divergence as $\epsilon \rightarrow 0$, and that the form of the leading term is controlled by a single coefficient for a conformal field theory [16-18]. It is known that the leading term does not actually take part in controlling the transformation of the surface operator under scaling or translation [16]. This follows from Gauss law when we consider integrating the stress tensor over an appropriate Gauss surface wrapping the surface operator. We are therefore most interested in the sub-leading terms in $\epsilon$. We make the working assumption that the sub-leading divergent terms can in fact be constructed using local geometric data-such as extrinsic curvatures and torsion- of the surface on which the operator wraps. This approach was taken in [19] where the authors there attempted a systematic study of OPE's of Wilson line operators in four dimensions, which is the classic example of extended operators. For simplicity, we focus on the co-dimension two operators in $d=3$ theories, so that they are essentially line operators, much like the Wilson line operators. We demonstrate however that our working assumption necessarily breaks down- that it is not possible to satisfy 
stress energy conservation if we insist upon constructing the sub-leading terms out of local geometric quantities. We check our claims by comparing our results with a holographic calculation of the stress tensor in the presence of a surface operator by inserting probes in the bulk. The holographic calculation produces an expectation of the stress tensor that is consistent with stress energy conservation. We demonstrate however that it is not expressible in terms of local geometric data, giving support to our field theoretic analysis.

An outline of our discussion is as follows: we begin a review of the Ward identities of twist operators in section 2, and then work out a systematic expansion of the expectation value of the stress tensor in the vicinity of a line operator in $2+1$ dimensions as a power series expansion in the infinitesimal perpendicular distance $\epsilon$, assuming that the terms are local geometric covariants. We obtain constraints of the coefficients of these terms up to the first subleading order in $\epsilon$ using tracelessness and stress energy conservation. In section 3, we compare these constraints with the known results of a line operator wrapping a circle, and we find complete agreement, although we demonstrate why this program would begin to fail in the next sub-leading order in the $\epsilon$ expansion. In section 4, we holographically compute the stress tensor induced by the presence of a line operator slightly deformed by a transverse wave from a straight line, and thus find evidence that supports our claim of a non-local stress tensor expansion. We conclude our paper with a few final comments in section 5. Some details of the calculations are relegated to the appendices.

\section{Ward identities and OPEs}

As shown in [6], under diffeomorphism, the entanglement entropy which is a special instance of a co-dimension two twist operator insertion, changes as follows:

$$
S_{\mathrm{EE}}(A, f * g)-S_{\mathrm{EE}}(A, g)=\int d x^{n+1} \sqrt{g} \xi_{\mu} \nabla_{\nu}\left(-\frac{2}{\sqrt{g}} \frac{\delta S_{\mathrm{EE}}(A, g)}{\delta g_{\mu \nu}}\right)
$$

where $f$ is a time-independent spatial diffeomorphism (generated by $\xi_{\mu}$ ) continuously connected to the identity, and $A$ denotes the region enclosed by the entangling surface which the inserted operator wraps around. Since

$$
S_{\mathrm{EE}}(f(A), g)=S_{\mathrm{EE}}(A, f * g),
$$

where $f * g$ denotes the pull back of the metric, one can replace a smooth deformation of the entangling surface by diffeomorphism.

The above is equivalent to [6]

$$
\nabla_{\nu}\left(-\frac{2}{\sqrt{g}} \frac{\delta S_{\mathrm{EE}}(A, g)}{\delta g_{\mu \nu}}\right)=F n^{\mu} \delta_{\Sigma}
$$

where $\Sigma$ is the entangling surface, and $F$ is some general function which depends on coordinates tangential to $\Sigma$. The unit vector $n^{\mu}$ is normal to the entangling surface and is in the radial direction of the co-dimension two plane. The l.h.s. of (2.3) is the diver- 
gence of the expectation value of the stress tensor in the presence of the twist operator. ${ }^{1}$ This is the statement that the twist operator inserted over $\Sigma$ breaks energy-momentum conservation. However, the r.h.s. of (2.3) has support only on the entangling surface so that the stress tensor conservation holds away from the entangling surface. Based on the ansatz consistent with the form of r.h.s. of (2.3), we will exploit the conservation law of stress tensor to extract the OPE of the stress tensor with the twist operator inserted on the entangling surface of general shape. We note that the information of $F$ is encoded in the OPE coefficients via Gauss's law.

\subsection{Constructing expectation value of the stress tensor relating to OPE}

From the discussion above, it is clear that at the end of the day, it is the UV divergent terms in the stress tensor expectation value that carry information about the functions $F$. Therefore, to extract this information for more general entangling surfaces, we would like to expand the stress tensor in powers of UV-cutoff $|\epsilon|$, i.e., the separation between the location of the stress tensor and the entangling surface. The expansion coefficients are then related to OPE coefficients of stress tensor with the surface operator inserted at the entangling surface.

To simplify the discussion and be concrete, we will focus on $d=3$, so that the entangling surface would be one dimensional.

The expansion of the stress tensor should take the following form,

$$
T_{\mu \nu}(x)=\frac{1}{|\epsilon|^{3}}\left(A_{\mu \nu}^{0}+|\epsilon| A_{\mu \nu}^{1}+\cdots\right) .
$$

Following the philosophy in [19], we assume the OPE coefficients $A_{\mu \nu}^{i}$ can be expressed in terms of local geometry data and use dimensionality as well as parity symmetry $(\sigma \rightarrow-\sigma$, where $\sigma$ parameterizes the curve) to write down their most general forms. Thus, the leading term should be as follows:

$$
A_{\mu \nu}^{0}=a_{1} \delta_{\mu \nu}+a_{2} t_{\mu} t_{\nu}+a_{3} \hat{n}_{\mu} \hat{n}_{\nu}
$$

where $t^{\mu}(\hat{x})$ is the unit tangent vector on the entangling surface evaluated at the point $\hat{x}$, and $\hat{n}$ is again the unit normal vector defined by

$$
\hat{n}_{\mu}=\frac{(x-\hat{x})_{\mu}}{\epsilon} .
$$

Some useful identities regarding the local geometrical quantities are relegated into the appendix A.

Similarly, by using the dimensional analysis and parity symmetry, we can build $A_{\mu \nu}^{1}$ out of the local geometric data as follows

$$
A_{\mu \nu}^{1}=K^{1}\left(b_{1} \delta_{\mu \nu}+b_{2} t_{\mu} t_{\nu}+b_{3} \hat{n}_{\mu} \hat{n}_{\nu}\right)+b_{4}\left(\hat{n}_{\mu} n_{\nu}^{a} K^{a}+\hat{n}_{\nu} n_{\mu}^{a} K^{a}\right) .
$$

\footnotetext{
${ }^{1}$ More precisely, we have $\frac{\delta S_{\mathrm{EE}}}{\delta g}=\lim _{n \rightarrow 1} \frac{1}{n-1} \frac{\delta \log Z_{n}}{\delta g}$ and the variation of $S_{\mathrm{EE}}$ gives the $\mathcal{O}(n-1)$ part of the actual stress with the twist operator inserted (the latter of which follows from the variation of $\log Z_{n}$, partition function with twist operator). But this discrepancy is relevant for neither the study of conservation nor locality.
} 
We note that the rule is that $\hat{n}$ and its derivatives thereof are allowed to appear an odd number of times at each order of the expansion, whereas $t^{\mu}$ and $k$ must appear an even number of times, to preserve the symmetry of the problem.

To continue, we also have

$$
\begin{aligned}
A_{\mu \nu}^{2}= & c_{0} \partial_{\sigma} K^{1} n_{(\mu}^{1} t_{\nu)}+c_{0}^{\prime} \partial_{\sigma} K^{a} n_{(\mu}^{a} t_{\nu)}+\left(K^{1}\right)^{2}\left(c_{1} \delta_{\mu \nu}+c_{2} t_{\mu} t_{\nu}+c_{3} n_{\mu}^{1} n_{\nu}^{1}\right) \\
& +c_{4} K^{1}\left(n_{\mu}^{1} n_{\nu}^{a} K^{a}+n_{\nu}^{1} n_{\mu}^{a} K^{a}\right)+c_{5} n_{\mu}^{a} n_{\nu}^{b} K^{a} K^{b} \\
& +\left(K^{a}\right)^{2}\left(c_{1}^{\prime \prime} \delta_{\mu \nu}+c_{2}^{\prime \prime} t_{\mu} t_{\nu}+c_{3}^{\prime \prime} \hat{n}_{\mu} \hat{n}_{\nu}\right) .
\end{aligned}
$$

We note that these parameters, $\left\{a_{i}, b_{i}, c_{i}, \cdots\right\}$ are not independent, since the stress tensor is conserved anywhere away from the entangling surface, and moreover in a conformal theory it has to be traceless. We can reduce the number of independent coefficients using these constraints, order by order in perturbation theory.

Plug in the expansion of $T_{\mu \nu}$ and one can obtain

$$
\partial^{\mu} T_{\mu \nu}=\frac{-3}{|\epsilon|^{4}} n^{1 \mu}\left(A_{\mu \nu}^{0}+|\epsilon| A_{\mu \nu}^{1}+\cdots\right)+\frac{1}{|\epsilon|^{3}}\left(\partial^{\mu} A_{\mu \nu}^{0}+n^{1 \mu} A_{\mu \nu}^{1}+|\epsilon| \partial^{\mu} A_{\mu \nu}^{1}+\cdots\right) .
$$

Furthermore,

$$
\partial^{\mu} A_{\mu \nu}^{0}=a_{2} \frac{n_{\nu}^{1} K^{a}}{1-|\epsilon| K^{1}}+\frac{a_{3} n_{\nu}^{1}\left(2-\frac{1}{\left(1-|\epsilon| K^{1}\right)}\right)}{|\epsilon|}
$$

and that

$$
\begin{aligned}
\partial^{\mu} A_{\mu \nu}^{1}= & \left(\partial_{\sigma} K^{1}-K^{a} n_{\beta}^{a} \partial_{\sigma} n^{1 \beta}\right)\left(b_{1}+b_{2}\right) s_{\nu}+\frac{1}{|\epsilon|} b_{1}\left(K^{a} n_{\nu}^{a}-K^{1} n_{\nu}^{1}\right) \\
& +K^{1}\left(\frac{b_{3} n_{\nu}^{1}}{|\epsilon|}\left(2-\frac{1}{1-|\epsilon| K^{1}}\right)+b_{2} \frac{n_{\nu}^{a} K^{a}}{1-|\epsilon| K^{1}}\right) \\
& +b_{4}\left(\frac{n_{\nu}^{a} K^{a}}{|\epsilon|}\left(2-\frac{1}{1-|\epsilon| K^{1}}\right)+\frac{K^{a}}{|\epsilon|}\left(n_{\nu}^{a}-\delta^{a 1} n_{\nu}^{1}-t_{\alpha} s^{\alpha} n_{\nu}^{1}\left(K^{a}\right)^{2}\right)\right)
\end{aligned}
$$

where $s_{\mu}=\frac{t_{\mu}}{1-|\epsilon| K^{1}}$ as defined in (A.7).

Now collecting all the results and requiring energy-momentum conservation $\partial^{\mu} T_{\mu \nu}=0$, at the leading order of $\epsilon$-expansion of (2.9), i.e., $\mathcal{O}\left(|\epsilon|^{-4}\right)$, we have

$$
3 a_{1}+2 a_{3}=0 \text {. }
$$

To the first sub-leading order, there are two independent terms whose coefficients we set to zero:

$$
-n_{\nu}^{1} K^{1}\left(3 b_{1}+b_{3}+d b_{4}+a_{3}\right)+n_{\nu}^{a} K^{a}\left(a_{2}+b_{1}\right)=0,
$$

giving

$$
3 b_{1}+b_{3}+3 b_{4}+a_{3}=0, \quad a_{2}+b_{1}=0 .
$$

Including constraints from tracelessness, we have in addition

$$
3 a_{1}+a_{2}+a_{3}=0, \quad 3 b_{1}+b_{2}+b_{3}+2 b_{4}=0 .
$$

In the next section we will check these relations by the known result of a spherical twist operator, and then consider more general surface operators. 


\section{Checking the circle in a conformal field theory}

In a conformal theory, we can determine the stress tensor in the presence of a spherical twist operator inserted along the spherical entangling surface. That is simply obtained by conformal transformation from the space $H_{d-1} \times S^{1}$ via

$$
\frac{R-\omega}{R+\omega}=\exp (-\sigma)
$$

where $\omega=r+i t$ is the complex coordinate made of the radial and Euclidean time coordinates $(r, t)$ in the flat frame, and $\sigma=u+i \tau$ denotes the hyperbolic radial coordinate in $H_{d-1}$ and Euclidean time coordinate of $S^{1}$ respectively.

In the $H_{d-1} \times S^{1}$ frame, purely from symmetry one can deduce that the stress tensor is diagonal, taking the following form:

$$
T_{\tau \tau}=-(d-1) P, \quad T_{A B}=P g_{A B},
$$

where $g_{A B}$ denotes the metric of $H_{d-1}$

Following through the conformal transformation, the stress tensor in the flat frame takes the following form:

$$
\begin{aligned}
T_{t t} & =\frac{(2 R)^{d} P}{4\left|R^{2}-\omega^{2}\right|^{d}}\left[2(2-d)-d\left(\frac{R^{2}-\omega^{2}}{R^{2}-\bar{\omega}^{2}}+\frac{R^{2}-\bar{\omega}^{2}}{R^{2}-\omega^{2}}\right)\right], \\
T_{x_{i} x_{j}} & =\frac{(2 R)^{d} P}{4\left|R^{2}-\omega^{2}\right|^{d}}\left[\left(2(2-d)+d\left(\frac{R^{2}-\omega^{2}}{R^{2}-\bar{\omega}^{2}}+\frac{R^{2}-\bar{\omega}^{2}}{R^{2}-\omega^{2}}\right)\right) \frac{x_{i} x_{j}}{r^{2}}+4\left(\delta_{i j}-\frac{x_{i} x_{j}}{r^{2}}\right)\right], \\
T_{t r} & =i\left|\frac{2 R}{R^{2}-\omega^{2}}\right|^{d} \frac{d P}{4}\left(\frac{R^{2}-\bar{\omega}^{2}}{R^{2}-\omega^{2}}-\frac{R^{2}-\omega^{2}}{R^{2}-\bar{\omega}^{2}}\right) .
\end{aligned}
$$

These expressions can be expanded in $\epsilon$, where we take

$$
\omega=R+(\delta r+i \delta t), \quad|\epsilon|^{2}=\delta r^{2}+\delta t^{2},
$$

from which we can extract the values of $\left\{a_{i}, b_{i}\right\}$ and so on defined in the previous section at least for $d=3$.

A useful expression to make the comparison is that the extrinsic curvatures are given by

$$
K^{1}=-\frac{\delta r}{R|\epsilon|}, \quad n_{\mu}^{a} K^{a}=-\left.\frac{x_{i}}{R^{2}}\right|_{x^{2}=R^{2}} .
$$

This allows us to evaluate the expectation value of the stress tensor. In particular,

$$
A_{t t}^{1}=\frac{-\delta r}{R|\epsilon|}\left(b_{1}+b_{3} \frac{\delta t^{2}}{\left|\epsilon^{2}\right|}\right), \quad A_{r r}^{1}=-\frac{\delta r}{R|\epsilon|}\left(b_{1}+b_{3} \frac{\delta r^{2}}{\epsilon^{2}}+2 b_{4}\right) .
$$

This can be compared with the corresponding expansion of the stress tensor

$$
\begin{aligned}
T_{t t} & =\frac{P}{|\epsilon|^{d+2}}\left(\left(\delta t^{2}-2 \delta r^{2}\right)+3 \delta r \frac{\left(\delta t^{2}+2 \delta r^{2}\right)}{2 R}\right)+\cdots \\
T_{r r} & =\frac{P}{|\epsilon|^{d+2}}\left(\left(\delta r^{2}-2 \delta t^{2}\right)-3 \delta r \frac{\delta r^{2}}{2 R}\right)+\cdots
\end{aligned}
$$


Comparing the two expressions, we get

$$
\begin{array}{ll}
a_{1}=-2 P, & a_{3}=3 P=a_{2} \\
b_{1}=-3 P, & b_{3}=\frac{3}{2} P=b_{4},
\end{array}
$$

We note that all the constraints (2.12) (2.14) (2.15) following from tracelessness and momentum conservation are satisfied as expected. We also note that these coefficients are over-determined and so provides a non-trivial check of the current construction.

\subsection{Hitting a rock - Failing Ward identity by local OPE for general entan- gling surfaces}

Despite that we have some success applying the ansatz to spherical entangling surface, challenge comes immediately when we try to deal with a less special surface $\Sigma$ in $2+1$ dimensions. As shown below the stress tensor from the ansatz for a general surface $\Sigma$ is not always conserved, nor does it provide the correct entanglement entropy perturbatively.

\subsubsection{Evidence 1}

In 2.1 we consider the energy-momentum conservation up to $\mathcal{O}\left(|\epsilon|^{-3}\right.$ and it yields the constraints (2.12)-(2.14). Now we will see that the conservation cannot hold at the order $\mathcal{O}\left(|\epsilon|^{-2}\right)$. At this order, the terms contributing to $\partial^{\mu} T_{\mu \nu}$ are from the order $\mathcal{O}\left(|\epsilon|^{0}\right)$ terms of

$$
\partial^{\mu} A_{\mu \nu}^{1}-n^{1 \mu} A_{\mu}^{2}+|\epsilon| \partial^{\mu} A_{\mu \nu}^{2} .
$$

As we can see from (2.11), the terms at this order from $\partial^{\mu} A_{\mu \nu}^{1}$ are ,

$$
\left.\partial^{\mu} A_{\mu \nu}^{1}\right|_{\mathcal{O}\left(|\epsilon|^{0}\right)}=\left(b_{1}+b_{2}\right) s_{\nu} \partial_{\sigma} K^{1}+\mathcal{O}\left(K^{a}\right)^{2}
$$

where the first term is proportional to $t_{\nu}$ and is linear in $\partial_{\sigma} K^{1}$, and the other terms involve $\left(K^{a}\right)^{2}$ but not $\partial_{\sigma} K^{1}$. On the other hand, from the defining equation (2.8) the terms in $\left.n^{1 \mu} A_{\mu \nu}^{2}\right|_{\mathcal{O}\left(|\epsilon|^{0}\right)}$ involving $\partial_{\sigma} K^{1}$ are instead in the form of $n^{1 \mu} \partial_{\sigma} K^{1}$ which cannot compensate the term in (3.14).

We note that in principle $\left.|\epsilon| \partial^{\mu} A_{\mu \nu}^{2}\right|_{\mathcal{O}\left(|\epsilon|^{0}\right)}$ can also provide terms proportional to $\partial_{\sigma} K^{1} t_{\mu}$. However as we can show explicitly that

$$
\begin{aligned}
\left.|\epsilon| \partial^{\mu} A_{\mu \nu}^{2}\right|_{\mathcal{O}\left(|\epsilon|^{0}\right)=} & 2 c_{1} K^{1}\left(K^{a} n_{\nu}^{a}-K^{1} n_{\nu}^{1}\right)+\left(K^{1}\right)^{2}\left(c_{3} n_{\nu}^{1}+c_{2} n_{\nu}^{a} K^{a}\right) \\
& +c_{4} K^{1}\left[2 n_{\nu}^{a} K^{a}-n_{\nu}^{1} K^{1}-t_{\alpha} s^{\alpha} n_{\nu}^{1}\left(K^{a}\right)^{2}\right]+c_{4}\left[\left(K^{a}\right)^{2}-\left(K^{1}\right)^{2}\right] n_{\nu}^{1} \\
& +2 c_{1}^{\prime \prime} K^{1}\left(K^{a} n_{\nu}^{a}-K^{1} n_{\nu}^{1}\right)-2 c_{1}^{\prime \prime} K^{1}\left(K^{a} n_{\nu}^{a}-K^{1} n_{\nu}^{1}\right) \\
& +\left(K^{a}\right)^{2}\left(c_{3}^{\prime \prime} n_{\nu}^{1}+c_{2}^{\prime \prime} n_{\nu}^{a} K^{a}\right)
\end{aligned}
$$

where we have used the identities in appendix A. We find no term in the form of $\partial_{\sigma} K^{1} t_{\mu}$ that can be used to cancel the term in (3.14). Moreover, after a detailed check we find that the other terms not involving $t_{\mu} \partial_{\sigma} K^{1}$ will all be canceled out exactly at this order.

As a result, the conservation of stress tensor at this order at least requires

$$
b_{1}+b_{2}=0,
$$


which is not satisfied for the known $b_{1}, b_{2}$ obtained from the case of spherical entangling surface (3.11). Since the coefficients are numbers that are independent of the shape, it follows that the stress tensor from our ansatz is not conserved unless the entangling surface satisfies $\partial_{\sigma} K^{1}=0$. This is consistent with the fact that the stress tensor for spherical entangling surface is conserved as discussed previously because $\partial_{\sigma} K^{1}=0$ in this case even though (3.16) is violated.

\subsubsection{Evidence 2}

In [12], it was shown that the entanglement entropy due to the deformation of a circular entangling surface (at $d=3$ ) does not receive linear correction from the deformation. Instead, the quadratic correction is non-zero and takes the form

$$
\Delta S_{\mathrm{EE}} \sim \epsilon^{2} n\left(n^{2}-1\right)\left(a_{n}^{2}+b_{n}^{2}\right),
$$

for the deformation in the form of

$$
\delta r \sim \epsilon\left(a_{n} \cos n \theta+b_{n} \cos n \theta\right)
$$

where $(r, \theta)$ are the polar coordinates parametrizing the disk region bounded by the entangling circle.

Similar contributions can be done for the deformation of a straight-line entangling surface. As we will show from the holographic calculations in the next section, the result is

$$
\delta S_{\mathrm{EE}} \sim \epsilon^{2} k^{3}\left(a_{k}^{2}+b_{k}^{2}\right),
$$

for the deformation taking the form

$$
\delta y=\epsilon\left(a_{k} \cos k x+b_{k} \sin k x\right)
$$

to an entangling surface aligned along the $x$ direction.

We note that if this result is obtainable from the OPE of the stress tensor with the line operator, it has to come from $A_{\mu \nu}^{2}$ as the linear order correction from $A_{\mu \nu}^{1}$ vanishes. Namely, consider an entangling surface only slightly deformed from a flat entangling surface. The deformation is given precisely as a wave above. In this case, the extrinsic curvature of this surface must take the form

$$
K \sim \epsilon k^{2}\left(a_{k} \cos k x+b_{k} \sin k x\right) .
$$

Now if we further perturb by another $\delta y$ of exactly the same waveform, then we can use (2.1) to compute the perturbation to $S_{\mathrm{EE}}$ to quadratic order in $\epsilon$. The only term that can contribute must be terms involving $\partial_{x} K \sim \epsilon k^{2}\left(-a_{k} \sin k x+b_{k} \cos k x\right)$.

The final form of the surface integral of the stress tensor would have the term

$$
\int A_{\mu \nu}^{2} n^{\mu} \xi^{\nu} \sim \int d x \partial_{x} K^{1}(\xi \cdot t)+\mathcal{O}\left(\epsilon^{3}\right)
$$

which unfortunately vanishes at the order of $\mathcal{O}\left(\epsilon^{2}\right)$ because $(\xi \cdot t) \sim \mathcal{O}\left(\epsilon^{2}\right)$. Moreover, there are no other $\mathcal{O}\left(\epsilon^{2}\right)$ terms from $\int A_{\mu \nu}^{2} n^{\mu} \xi^{\nu}$ which are local and dependent only on the 
geometry of the entangling surface. In a word, the stress tensor that follows from the OPE ansatz does not reproduce the known form of perturbed entanglement entropy because it predicts $\delta S_{\mathrm{EE}}=0$ up to second order in shape deformation.

This appears to be strong evidence that the OPE coefficients of the stress tensor must contain non-local terms, in addition to the terms we have found, despite the fact that the explicit results of a spherical entangling surface satisfies all the constraints following from our local construction of the divergent terms.

\section{Holographic calculation}

In this section we will re-consider the issues discussed in sec 3 in the holographic dual. Especially we will derive the holographic stress tensor in the presence of a (one dimensional and possibly wavy) twist operator at $\mathrm{d}=3$, and find that it is conserved. The result implies that the OPE of the stress tensor with the twist operator at $d=3$ cannot be in the local expression of geometric quantities as extrinsic curvatures and their derivatives.

\subsection{Planar entangling surface perturbed}

We first consider the variation of the holographic entanglement entropy due to the deformation of the flat entangling surface at $d=3$. This is an analogy to what has been done in [12] for the deformation of the spherical entangling surface.

We choose the following metric for the dual $\mathrm{AdS}_{4}$.

$$
\mathrm{d} s^{2}=\frac{L^{2}}{z^{2}}\left(d t^{2}+d z^{2}+d x^{2}+d y^{2}\right)
$$

The entangling surface lies at $y=0($ also $t=0)$ and we consider the following perturbation

$$
\delta y_{0}=\epsilon e^{i k x}
$$

for very small $\epsilon$.

We can take the following ansatz for the corresponding minimal surface

$$
y(x, z)=y_{1}(z) \delta y_{0},
$$

and also impose the following boundary conditions to conform to (4.2) at $z=0$, i.e.,

$$
y_{1}(0)=1, \quad y_{1}(\infty)=0 .
$$

The minimal surface is obtained by minimizing the area

$$
A=\int \frac{\sqrt{\left(1+\left|\partial_{x} y\right|^{2}\right)\left(1+\left|\partial_{z} y\right|^{2}\right)-\left|\partial_{x} y \partial_{z} y\right|^{2}}}{z^{2}} d x d z
$$

where we include the complex conjugate to make the area real. The change of the area of the minimal surface due to the deformation (4.4) is then given by

$$
\Delta A=\epsilon^{2} \int d x \int_{\mu}^{\infty} d z \frac{\left(\partial_{z} y_{1}\right)^{2}+k^{2} y_{1}^{2}}{2 z^{2}}
$$


from which we get the equation of motion for $y_{1}(z)$

$$
z \partial_{z}^{2} y_{1}-2 \partial_{z} y_{1}-k^{2} z y_{1}=0
$$

Imposing the boundary condition (4.4), we obtain the special solution (assuming $k>0$ for simplicity),

$$
y_{1}(z)=(k z+1) e^{-k z} .
$$

Substituting (4.8) into (4.6), we get the change of the holographic entanglement entropy

$$
\Delta S_{\mathrm{EE}} \sim \Delta A=\epsilon^{2} \frac{k^{2}(k \mu+1) e^{-2 k \mu}}{2 \mu} \int d x=\epsilon^{2}\left(\frac{k^{2}}{2 \mu}-\frac{k^{3}}{2}+\mathcal{O}(\mu)\right) \int d x
$$

where $\mu$ is the IR cutoff of the bulk as introduced in (4.6). This result is very similar to (44) in [12]. Moreover, it is nonzero in contrast to the null result from the OPE of stress tensor with twist operator.

\subsection{Holographic computation of the stress tensor}

In the previous consideration we can obtain the change of the holographic entanglement entropy, but did not obtain the corresponding holographic stress tensor in the presence of the wavy twist operator, which is the holographic dual to the Ryu-Takayanagi (RT) action of the minimal surface. Now we would like to derive such a stress tensor holographically and check its conservation directly. The way to do it is to introduce a (probing) boundary source metric variation, which then back-reacts to the bulk metric. We then evaluate the total on-shell bulk action, including the RT one $[20,21]$ which represents the insertion of the entangling surface on the dual field theory side. That is, the total bulk action is $S_{\text {total }}:=n S_{\mathrm{gr}}+(n-1) S_{\mathrm{EE}}$ where $S_{\mathrm{gr}}$ is the bulk gravity action and $S_{\mathrm{EE}}$ is the RT one. Taking the variation of the on-shell action w.r.t. the boundary source metric variation and then setting the source to zero, we will obtain the stress tensor in the presence of the entangling surface inserted as a twist operator, i.e., the OPE of stress tensor with twist operator.

We will not include the back reaction of the surface operator on the background, presumably because by taking the replica index $n$ to one the dimension of the twist operator approaches zero, or equivalently in the holographic bulk its coupling to the background geometry is suppressed by $n-1$. Since the extremal surface satisfies the equation of motion following from the Ryu-Takayanagi action, to linear order in the metric perturbation, we need only to evaluate the action on-shell neither back-reacting the metric perturbation nor the extremal surface.

We choose the Poincare coordinates for the AdS space, and the deformed metric due to the boundary source metric perturbation takes the following form,

$$
d s^{2}=\frac{1}{z^{2}}\left[\left(1+h_{z z}\right) d z^{2}+\left(\eta_{\mu \nu}+h_{\mu \nu}\right) d x^{\mu} d x^{\nu}+2 h_{z \mu} d z d x_{\mu}\right]
$$


We are following gauge choice in [22], in which

$$
\begin{aligned}
h_{i j} & =c_{d} \int d^{d} x^{\prime} \frac{z^{d-2}}{f^{d}} P_{i j a b} \delta G_{a b} \\
h_{z i} & =c_{d} \frac{d}{d-1} \int d^{d} x^{\prime} \frac{z^{d-3}}{f^{d-1}} B_{i a b} \delta G_{a b} \\
h_{z z} & =-c_{d} \frac{d}{d-1} \int d^{d} x^{\prime} \frac{z^{d-2}}{f^{d}} C_{a b} \delta G_{a b},
\end{aligned}
$$

where

$$
f=z^{2}+\hat{x}_{\mu} \hat{x}^{\mu}, \quad \hat{x}^{\mu}:=x^{\mu}-x^{\prime \mu},
$$

and

$$
\begin{aligned}
& c_{d}=\frac{\Gamma(d)}{\pi^{\frac{d}{2}} \Gamma\left(\frac{d}{2}\right)}, \\
& B_{i a b}=\frac{1}{4} \partial_{i} J_{j k}(\hat{x}) P_{j k a b}, \quad C_{a b}=J_{i j}(\hat{x}) \\
& J_{\mu \nu}(\hat{x})=\delta_{\mu \nu}-2 \frac{\hat{x}_{\mu} \hat{x}_{\nu}}{x^{2}}, \quad P_{i j a b}=\frac{1}{2}\left(\delta_{i a} \delta_{j b}+\delta_{i b} \delta_{j a}\right),
\end{aligned}
$$

and $\delta G_{\mu \nu}$ is the (probing) source metric variation at the boundary. We note that the expectation value of the boundary stress tensor is given by

$$
\left\langle T^{\mu \nu}\right\rangle_{\Sigma}=\left.\left.\frac{\delta S_{\text {total }}}{\delta G_{\mu \nu}}\right|_{\delta G_{\mu \nu} \rightarrow 0} \simeq \frac{\delta S_{\mathrm{EE}}}{\delta G_{\mu \nu}}\right|_{\delta G_{\mu \nu} \rightarrow 0}
$$

In the above, $\simeq$ means that we take $n \longrightarrow 1$ limit to suppress the back reaction to the bulk metric.

We will focus on the case in which the entangling surface is almost planar (perturbed by (4.2)), inserted at $t:=x_{1}=0$ and $y:=x_{d}=\epsilon e^{i k x}$. In this case the extremal surface in the bulk is described by (4.3) (4.8) and is translationally invariant along $x_{2}, \cdots x_{d-1}$.

In the following we will consider only $d=3$. We choose the static gauge such that the induced metric on extremal surface takes the form (up to the terms possibly contributing to $\mathcal{O}(\epsilon)^{2}$ part of $\left.\Delta S_{\mathrm{EE}}\right)$,

$$
\begin{aligned}
k_{a b} d \sigma^{a} d \sigma^{b}=\frac{1}{z^{2}}\left[\left(1+h_{z z}+\left(\partial_{z} y\right)^{2}\right.\right. & \left.+2 h_{z y} \partial_{z} y\right) d z^{2}+2 h_{x z} d x d z \\
& \left.+\left(1+h_{x x}+\left(\partial_{x} y\right)^{2}+2 h_{x y} \partial_{x} y\right) d x^{2}\right]+\cdots
\end{aligned}
$$

where we have included derivatives of $y$ w.r.t. the world-volume coordinates for latter convenience. For the strictly planar case, $y=0$ and therefore all terms involving $y$ actually vanishes.

To the lowest order in $\epsilon$, i.e., $\mathcal{O}(1)$, the stress tensor we get is that for a straight twist operator (see e.g. [17])

$$
\left\langle T_{\mu \nu}^{\mathcal{O}(1)}\right\rangle=-\frac{2}{8 \pi\left(t^{\prime 2}+y^{\prime 2}\right)^{3 / 2}}\left(\delta_{\mu \nu}-\frac{3}{2} t_{\mu} t_{\nu}-\frac{3}{2} \hat{n}_{\mu} \hat{n}_{\nu}\right)
$$

which by itself is conserved and traceless. 
The shape dependence of the stress tensor appears at the next leading order. Let us first consider the case in which the only nonvanishing component is $\delta G_{x y}$. The $\mathcal{O}(\epsilon)$ order contribution to the (change of the) stress tensor reads $\left(d=3, \hat{x}=x-x^{\prime}\right)$

$$
\begin{aligned}
& \left\langle T_{x y}{ }^{\mathcal{O}(\epsilon)}\right\rangle \\
& =\left.\frac{\delta}{\delta G_{x y}} \int d x d z\left(\frac{\sqrt{1+h_{z z}+2 i \epsilon k\left[h_{x y}+k z\left(h_{x y}+i h_{z y}\right)\right] e^{-k z+i k x}+\mathcal{O}(\epsilon)^{2}}}{z^{2}}-\frac{1}{z^{2}}\right)\right|_{\delta G_{x y} \rightarrow 0} \\
& =\epsilon \int d x d z \frac{z e^{i k x-k z}}{\pi^{2}\left(t^{\prime 2}+\hat{x}^{2}+y^{\prime 2}+z^{2}\right)^{5}}\left\{6 \hat{x}(k z+1)\left(t^{\prime 2}+\hat{x}^{2}-7 y^{\prime 2}+z^{2}\right)\right. \\
& +k\left(t^{\prime 2}+\hat{x}^{2}+y^{\prime 2}+z^{2}\right)\left[t^{\prime 2}(3 k \hat{x}+2 i k z+2 i)+3 k \hat{x}^{3}+2 i \hat{x}^{2}(k z+1)\right. \\
& \left.\left.+3 k \hat{x}\left(z^{2}-y^{\prime 2}\right)+2 i(k z+1)\left(y^{\prime 2}+z^{2}\right)\right]\right\} .
\end{aligned}
$$

In the above we have set $y(x, z)$ of the extremal surface into the form of (4.3) and (4.8). The integration over $x$ in (4.18) can be done but we only manage to do the $z$ integration after an expansion in momentum $k$. After some tedious calculation, we obtain the result in the following form,

$$
\left\langle T_{x y}^{\mathcal{O}(\epsilon)}\right\rangle=\epsilon e^{i k x^{\prime}}\left[\frac{3 i t^{\prime 2} k}{8 \pi\left(t^{\prime 2}+y^{\prime 2}\right)^{5 / 2}}-\frac{i\left(3 t^{\prime 2}+2 y^{\prime 2}\right) k^{3}}{16 \pi\left(t^{\prime 2}+y^{\prime 2}\right)^{3 / 2}}-\frac{i k^{4}}{8 \pi}+\mathcal{O}\left(k^{5}\right)\right] .
$$

The series in $k$ above can also be understood as OPE of stress tensor with the twist operator since $\delta \equiv\left(t^{\prime 2}+y^{\prime 2}\right)^{1 / 2}$ is the minimal distance from the location of $T_{\mu \nu}$ to the plane $(y=t=0)$, i.e., where the twist operator is centered.

The other components of the stress tensor in the presence of the twist operator can be worked out similarly, and the detailed forms are put in the appendix B. Based on the detailed form of the stress tensor listed in appendix B order by order in $k$-expansion, it is straightforward to show that the stress tensor is traceless and conserved, i.e.,

$$
\left\langle T_{\mu}^{\mu \mathcal{O}(\epsilon)}\right\rangle=0, \quad \partial^{\mu}\left\langle T_{\mu \nu}^{\mathcal{O}(\epsilon)}\right\rangle=0 .
$$

Note that unlike the tracelessness, the conservation of $\left\langle T_{\mu \nu} \mathcal{O}(\epsilon)\right\rangle$ is not easy to see without performing the integration along the extremal surface.

The particular form of the OPE coefficients seems to rule out the possibility that they can be expressed covariantly in terms of local geometric quantities. For example, we can consider the $\mathcal{O}\left(k^{2}\right)$ term in $\left\langle T_{y y} \mathcal{O}(\epsilon)\right\rangle$ (see (B.3)) and denote the coefficient as $a_{y y}^{(2)}$. Note that the relevant extrinsic curvature in this case is $K^{y}=k^{2} \epsilon e^{i k x}$ and none of $n_{\mu}^{1,2}, t_{\mu}$ (defined in appendix A) have any components proportional to $k^{2}$. In other words, $a_{y y}^{(2)}$ should contain one $K^{y}$. On the other hand, $a_{y y}^{(2)}$ is also proportional to $y^{3}$, or rather $\left(n_{y}^{1}\right)^{3}$ and does not appear to follow from any 2-tensor built from $n_{\mu}^{1,2}, t_{\mu}$ and $\delta_{\mu \nu}$.

With $\left\langle T_{\mu \nu}{ }^{\mathcal{O}(\epsilon)}\right\rangle$, one can recover the holographic entanglement entropy computed in sec 4.1. More explicitly, we consider perturbation of the entangling surface given by $\delta y=$ $\tilde{\epsilon} e^{i k x}$ (i.e., $\epsilon \rightarrow \epsilon+\tilde{\epsilon}$ ). With the following coordinate transformation

$$
y \rightarrow y-\tilde{\epsilon} e^{i k x}, \quad z \rightarrow z, \quad x \rightarrow x,
$$


the entangling surface can be turned back into its original shape $\left(y=\epsilon e^{i k x}\right)$. The resulting first order perturbation to $G_{\mu \nu}$ is

$$
\delta G_{x y}=-i k \tilde{\epsilon} e^{i k x}
$$

with all other components of $\delta G_{i j}$ being zero. This perturbation can also be understood as being generated by the vector $\xi$ whose only nonvanishing component is $\xi_{y}=-\tilde{\epsilon} e^{i k x} .^{2}$ Substituting in (4.16) and (4.22), we get the leading order correction to entanglement entropy,

$$
\begin{aligned}
\Delta S_{\mathrm{EE}}= & \int d^{3} x^{\prime}\left\langle T_{x y}{ }^{\mathcal{O}(\epsilon)}\left(x_{\mu}^{\prime}\right)\right\rangle \delta G_{x y}^{*}\left(x_{\mu}^{\prime}\right) \\
= & \epsilon \tilde{\epsilon} \int d^{3} x^{\prime} \int d x d z\left[6 i k \hat { x } z \left(-\frac{2}{\pi^{2} f^{5}} 2(k z+1)\left(t^{\prime 2}+\hat{x}^{2}-7 y^{\prime 2}+z^{2}\right)\right.\right. \\
& \left.\left.\quad-\frac{1}{\pi^{2} f^{4}} k^{2}\left(t^{\prime 2}+\hat{x}^{2}-y^{\prime 2}+z^{2}\right)\right)+\frac{4 k^{2} z(k z+1)}{\pi^{2} f^{3}}\right] e^{i k \hat{x}-k z}+\mathcal{O}\left(\epsilon^{3}\right) .
\end{aligned}
$$

Note that $y(x, z)$ in $h_{z i}$ only gives contribution of higher orders in $\epsilon$ and therefore can be set to be zero. On the other hand, unlike $h_{z i}, h_{z z}$ is not multiplied by $\epsilon$ and therefore we need to expand $y \sim \mathcal{O}(\epsilon)$ in $f$ and $C_{x y}$. The integration of $y^{\prime}$ and $t^{\prime}$ can be performed straightforwardly and the result is

$$
\Delta S_{\mathrm{EE}}=\epsilon \tilde{\epsilon} \int d x^{\prime} \int d x d z k^{2} z\left(\frac{-2 i k \hat{x}}{\pi\left(\hat{x}^{2}+z^{2}\right)^{2}}+\frac{2(k z+1)}{\pi\left(\hat{x}^{2}+z^{2}\right)^{2}}\right) e^{i k \hat{x}-k z} .
$$

We notice that $h_{z z}$ actually has no contribution to $\delta S_{\mathrm{EE}}$. The integration of $x$ is essentially the Fourier transform and we get

$$
\begin{aligned}
\Delta S_{\mathrm{EE}} & =\epsilon \tilde{\epsilon} k^{2} \int_{\mu}^{\infty} d z e^{-2 k z}\left(k^{2}+\frac{(k z+1)^{2}}{z^{2}}\right) \int d x^{\prime} \\
& =\epsilon \tilde{\epsilon} k^{2} \frac{(1+k \mu) e^{-2 k \mu}}{\mu} \int d x^{\prime}=2 \epsilon \tilde{\epsilon}\left(\frac{k^{2}}{2 \mu}-\frac{k^{3}}{2}+\mathcal{O}(\mu)\right) \int d x^{\prime} .
\end{aligned}
$$

The result reproduces (4.9) exactly. To be more precise, we perform the replacement $\epsilon \rightarrow \epsilon+\tilde{\epsilon}$ in (4.9) and find the terms of the order of $\mathcal{O}(\epsilon \tilde{\epsilon})$ agree.

To summarize, the stress tensor in the presence of a wavy (co-dimension two) twist operator is computed holographically in $\mathrm{AdS}_{4}$. We found that the stress tensor in this case is conserved but its OPE coefficients is non-local. Similar conclusions can be obtained for a perturbed circular twist operator which is related to a wavy line operator by a conformal mapping.

\section{Conclusion}

In dimensions higher than two, a co-dimension two twist operator is a surface operator and the precise formulation of its OPE with some local conserved charge density such as

\footnotetext{
${ }^{2}$ Note that with an extra gauge transformation $\tilde{\xi}_{x}=i \tilde{\epsilon} k y e^{i k x}$, we can get to the metric in the normal coordinates used in [8] (which has $G_{x y}=0, G_{y y} \propto y K_{x x}^{y}$ ). This vector $\tilde{\xi}$ vanishes on the entangling surface $(y=0)$ and therefore the diffeomorphism it generates does not change the entanglement entropy.
} 
stress tensor have not been fully explored. As a starter, in this paper we study the OPE of stress tensor with a line twist operator in $2+1$ dimensions. We try an ansatz for such OPE coefficients constructed out of local geometric data encoding the shape of the line operator. It turns out that the resulting stress tensor in the presence of the twist operator can not be conserved for general (wavy) shapes of line operator except for highly symmetric one such as planar or spherical line operators. This is in contrast to the conserved holographic stress tensor, which we have constructed in this paper in the presence of the line twist operator based on RT-formulation. Furthermore, we demonstrated that the stress tensor obtained holographically is not expressible as local geometric expansion, implying that the OPE of stress tensor with line twist operator cannot be expressed in terms of local geometric data only. It is possible that the Ward identities could be anomalous in the presence of surface operators, however, the holographic results suggest otherwise.

The perturbative computation we perform in sec 4 is done holographically. It would be interesting to see whether the same stress tensor and entanglement entropy can be obtained from the integrated correlation functions of the CFT side using the second order perturbation (two insertions of stress tensors, see e.g. [8]). However, it should be noted that there might be some subtleties in pushing the perturbative computation to second order. For example, it remains unclear [11] how to reproduce the shape dependence of the entanglement entropy (in four dimensions) using this approach.

We should emphasize the failure of OPE in terms of local geometric data could be peculiar in odd dimensions. In contrast, the universal part of the entanglement entropy in four dimensions is known to have local expressions [23, 24] in terms of (Graham-Witten) conformal anomalies encoding geometric information of the twist operator, even though that does not constitute a proof that the OPE coefficients are local. It is not clear whether the fact that conformal anomaly does not exist in odd dimensions should make any further difference. Thus, it deserves further study of the issues raised in the current paper to get a clearer understanding of the results in both even and odd dimensions.

\section{Acknowledgments}

LYH is grateful to inspiring discussions with R. Myers and J. Gomis, A. Kapustin and particularly S. Matsuura who collaborated at the early state of the project. We also thank D. Gaiotto for inspiring email correspondence. FLL is supported by MoST grant: 1032112-M-003 -001 -MY3 and 103-2811-M-003 -024, he is also supported by NCTS north and thanks for discussions with Juinn-Wei Chen. The work of XH is supported by MoST grant: 103-2811-M-003-024.

\section{A Useful Identities regarding the local geometrical quantities}

To obtain further expansions, we would need a few more building blocks.

To begin with, we need a complete basis of vectors in the vicinity of $\hat{x}$, let us also define the vector

$$
k_{\mu}=\epsilon_{\mu \nu \rho} \hat{n}_{\nu} t_{\rho}
$$

One can readily check that $k_{\mu} k^{\mu}=1$ and by construction it is normal to both $\hat{n}_{\mu}$ and $t_{\mu}$. 
We can evaluate the extrinsic curvatures, defined as

$$
K^{a}=-t^{\mu} t^{\nu} \nabla_{\mu} n_{\nu}^{a}
$$

where $n^{a}, a=\{1,2\}$ denotes the two possible unit normals $n^{1}=\hat{n}$ and $n^{2}=k$ respectively.

One very useful identity is

$$
\frac{\partial \sigma}{\partial x^{\mu}}=\frac{t_{\mu}}{1-\hat{n}^{\alpha} \partial_{\sigma} t_{\alpha}},
$$

where $\sigma$ is a convenient parametrization of the entangling curve, such that it's trajectory in spacetime can be denoted by $\left\{y^{\mu}(\sigma)\right\}$, and that

$$
t^{\mu}=\mathcal{N} \frac{\partial y^{\mu}(\sigma)}{\partial \sigma}
$$

where the normalization factor $\mathcal{N}=1$ if $\sigma$ is chosen to be some affine parameter.

The extrinsic curvature is therefore given by

$$
K^{a}=n_{\nu}^{a} \partial_{\sigma} t^{\nu}
$$

for affine parameter $\sigma$.

This identity is derived by requiring that as the insertion point $x^{\mu}$ is varied, $\hat{x}\left(\sigma_{0}\right)$ should vary accordingly so that

$$
\hat{n}_{\mu} t^{\mu}=0
$$

remains true.

There are a few useful identities:

$$
\begin{array}{rlrl}
\partial_{\mu} n_{\nu}^{1} & =\frac{1}{|\epsilon|}\left(\delta_{\mu \nu}-t_{\mu} s_{\nu}-n_{\mu}^{1} n_{\nu}^{1}\right), & s_{\mu} & =\frac{\partial \sigma}{\partial x^{\mu}}=\frac{t_{\mu}}{1-|\epsilon| K^{1}} . \\
\partial_{\mu} n_{\nu}^{2} & =\frac{1}{|\epsilon|} \epsilon_{\nu}^{\alpha \beta}\left(\delta_{\mu \alpha} t_{\beta}-t_{\mu} s_{\alpha} t_{\beta}-n_{\mu}^{1} n_{\alpha}^{1} t_{\beta}\right)+\epsilon_{\nu}^{\alpha \beta} n_{\alpha}^{a} K^{a} s_{\mu} n_{\beta}^{1}, \\
\partial_{\mu}|\epsilon| & =n_{\mu}^{1}, \\
\partial_{\mu} K^{a} & =\partial_{\mu}\left(n_{\alpha}^{a} \partial_{\sigma} t^{\alpha}\right)=s_{\mu} K^{b} n^{a}{ }^{\beta} \partial_{\sigma} n_{\beta}^{a}+s_{\mu} \partial_{\sigma} K^{a}+K^{b} n^{b \beta} \partial_{\mu} n_{\alpha}^{a} \\
\partial_{\mu} t^{\nu} & =\frac{\partial^{2} y^{\nu}}{\partial \sigma^{2}} s_{\mu}=n_{\nu}^{a} K^{a} s_{\mu}, \\
\frac{\partial t_{\mu}}{\partial \sigma} & =t^{\alpha} \partial_{\alpha} t_{\mu}=n_{\nu}^{a} K^{a}, \\
\partial_{\sigma} n_{\nu}^{a} & =-K^{a} t_{\nu}, & \frac{\partial^{2} t_{\mu}}{\partial \sigma^{2}} & =t^{\alpha} \partial_{\alpha} t_{\mu}=\frac{n_{\nu}^{a} K^{a}}{1-|\epsilon| K^{1}} .
\end{array}
$$

\section{B Holographic stress tensor in the presence of twist operator}

Follow the same procedure in deriving $\left\langle T_{x y} \mathcal{O}(\epsilon)\right\rangle$, one can derive the other components of the stress tensor in the similar way. The final results are listed below by $k$-expansion and 
integrating out the worldvolume $\int d x \cdots$,

$$
\begin{gathered}
\left\langle T_{x y}{ }^{\mathcal{O}(\epsilon)}\right\rangle=\epsilon e^{i k x}\left[\frac{3 i t^{2} k}{8 \pi\left(t^{2}+y^{2}\right)^{5 / 2}}-\frac{i\left(3 t^{2}+2 y^{2}\right) k^{3}}{16 \pi\left(t^{2}+y^{2}\right)^{3 / 2}}-\frac{i k^{4}}{8 \pi}+\mathcal{O}\left(k^{5}\right)\right] \\
\left\langle T_{t y}{ }^{\mathcal{O}(\epsilon)}\right\rangle=\epsilon e^{i k x}\left[-\frac{3 t\left(t^{2}-4 y^{2}\right)}{8 \pi\left(t^{2}+y^{2}\right)^{7 / 2}}-\frac{3 t\left(t^{2}+2 y^{2}\right) k^{2}}{16 \pi\left(t^{2}+y^{2}\right)^{5 / 2}}+\frac{t k^{3}}{4 \pi\left(t^{2}+y^{2}\right)}\right. \\
\left.-\frac{t\left(9 t^{2}+8 y^{2}\right) k^{4}}{64 \pi\left(t^{2}+y^{2}\right)^{3 / 2}}+\mathcal{O}\left(k^{5}\right)\right]
\end{gathered}
$$

and

$$
\begin{aligned}
& \left\langle T_{x x}{ }^{\mathcal{O}(\epsilon)}\right\rangle=\epsilon e^{i k x}\left[\frac{3 y}{8 \pi\left(t^{2}+y^{2}\right)^{5 / 2}}-\frac{3 y k^{2}}{16 \pi\left(t^{2}+y^{2}\right)^{3 / 2}}+\frac{5 y k^{4}}{64 \pi \sqrt{t^{2}+y^{2}}}+\mathcal{O}\left(k^{5}\right)\right], \\
& \left\langle T_{t t}{ }^{\mathcal{O}(\epsilon)}\right\rangle=\epsilon e^{i k x}\left[\frac{3\left(3 t^{2} y-2 y^{3}\right)}{8 \pi\left(t^{2}+y^{2}\right)^{7 / 2}}+\frac{3 y\left(t^{2}+2 y^{2}\right) k^{2}}{16 \pi\left(t^{2}+y^{2}\right)^{5 / 2}}-\frac{y k^{3}}{4 \pi\left(t^{2}+y^{2}\right)}\right. \\
& \left.+\frac{y\left(7 t^{2}+6 y^{2}\right) k^{4}}{64 \pi\left(t^{2}+y^{2}\right)^{3 / 2}}+\mathcal{O}\left(k^{5}\right)\right], \\
& \left\langle T_{t x}^{\mathcal{O}(\epsilon)}\right\rangle=\epsilon e^{i k x}\left[-\frac{3 i t y k}{8 \pi\left(t^{2}+y^{2}\right)^{5 / 2}}+\frac{i t y k^{3}}{16 \pi\left(t^{2}+y^{2}\right)^{3 / 2}}+\mathcal{O}\left(k^{5}\right)\right],
\end{aligned}
$$

where we have dropped the primes of $t^{\prime}$ and $y^{\prime}$ for simplicity.

Open Access. This article is distributed under the terms of the Creative Commons Attribution License (CC-BY 4.0), which permits any use, distribution and reproduction in any medium, provided the original author(s) and source are credited.

\section{References}

[1] D. Gaiotto, A. Kapustin, N. Seiberg and B. Willett, Generalized global symmetries, JHEP 02 (2015) 172 [arXiv:1412.5148] [INSPIRE].

[2] P. Calabrese and J.L. Cardy, Entanglement entropy and quantum field theory, J. Stat. Mech. 0406 (2004) P06002 [hep-th/0405152] [INSPIRE].

[3] P. Calabrese and J.L. Cardy, Entanglement entropy and quantum field theory: a non-technical introduction, Int. J. Quant. Inf. 4 (2006) 429 [quant-ph/0505193] [INSPIRE].

[4] H. Casini, Entropy inequalities from reflection positivity, J. Stat. Mech. (2010) P08019 [arXiv: 1004.4599] [INSPIRE].

[5] B. Swingle, Mutual information and the structure of entanglement in quantum field theory, arXiv: 1010.4038 [INSPIRE]. 
[6] S. Banerjee, Wess-Zumino consistency condition for entanglement entropy, Phys. Rev. Lett. 109 (2012) 010402 [arXiv:1109.5672] [INSPIRE].

[7] M. Billó et al., Line defects in the 3D Ising model, JHEP 07 (2013) 055 [arXiv:1304.4110] [INSPIRE].

[8] V. Rosenhaus and M. Smolkin, Entanglement entropy: a perturbative calculation, JHEP 12 (2014) 179 [arXiv:1403.3733] [INSPIRE].

[9] V. Rosenhaus and M. Smolkin, Entanglement entropy flow and the Ward identity, Phys. Rev. Lett. 113 (2014) 261602 [arXiv:1406.2716] [INSPIRE].

[10] V. Rosenhaus and M. Smolkin, Entanglement entropy, planar surfaces and spectral functions, JHEP 09 (2014) 119 [arXiv:1407.2891] [INSPIRE].

[11] V. Rosenhaus and M. Smolkin, Entanglement entropy for relevant and geometric perturbations, JHEP 02 (2015) 015 [arXiv:1410.6530] [INSPIRE].

[12] A. Allais and M. Mezei, Some results on the shape dependence of entanglement and Rényi entropies, Phys. Rev. D 91 (2015) 046002 [arXiv: 1407.7249] [INSPIRE].

[13] M. Mezei, Entanglement entropy across a deformed sphere, Phys. Rev. D 91 (2015) 045038 [arXiv: 1411.7011] [INSPIRE].

[14] D.E. Berenstein, R. Corrado, W. Fischler and J.M. Maldacena, The operator product expansion for Wilson loops and surfaces in the large- $N$ limit, Phys. Rev. D 59 (1999) 105023 [hep-th/9809188] [INSPIRE].

[15] J. Gomis and T. Okuda, S-duality, 't Hooft operators and the operator product expansion, JHEP 09 (2009) 072 [arXiv:0906.3011] [INSPIRE].

[16] A. Kapustin, Wilson-'t Hooft operators in four-dimensional gauge theories and S-duality, Phys. Rev. D 74 (2006) 025005 [hep-th/0501015] [InSPIRE].

[17] L.-Y. Hung, R.C. Myers, M. Smolkin and A. Yale, Holographic calculations of Rényi entropy, JHEP 12 (2011) 047 [arXiv:1110.1084] [INSPIRE].

[18] L.-Y. Hung, R.C. Myers and M. Smolkin, Twist operators in higher dimensions, JHEP 10 (2014) 178 [arXiv:1407.6429] [INSPIRE].

[19] T. Azuma and H. Kawai, OPE between the energy momentum tensor and the Wilson loop in $N=4$ super Yang-Mills theory, Prog. Theor. Phys. 106 (2001) 255 [hep-th/0106063] [INSPIRE].

[20] S. Ryu and T. Takayanagi, Holographic derivation of entanglement entropy from AdS/CFT, Phys. Rev. Lett. 96 (2006) 181602 [hep-th/0603001] [INSPIRE].

[21] S. Ryu and T. Takayanagi, Aspects of holographic entanglement entropy, JHEP 08 (2006) 045 [hep-th/0605073] [INSPIRE].

[22] H. Liu and A.A. Tseytlin, $D=4$ super Yang-Mills, $D=5$ gauged supergravity and $D=4$ conformal supergravity, Nucl. Phys. B 533 (1998) 88 [hep-th/9804083] [INSPIRE].

[23] S.N. Solodukhin, Entanglement entropy, conformal invariance and extrinsic geometry, Phys. Lett. B 665 (2008) 305 [arXiv: 0802.3117] [InSPIRE].

[24] A. Schwimmer and S. Theisen, Entanglement entropy, trace anomalies and holography, Nucl. Phys. B 801 (2008) 1 [arXiv:0802.1017] [INSPIRE]. 